\title{
Physicist meets chemist
}

\author{
Changxu Liu and Jianfeng Huang reflect on their experiences of a collaborative research project that was \\ at a crossroads between physics and chemistry.
}

Changxu: Throughout my $\mathrm{PhD}$, I have always had a strong appreciation for how the natural world can inspire the development of new photonic devices. Millions of years of evolution have led to a range of fascinating optical phenomena. Butterfly wings are, for example, covered with periodic microstructures that enhance the reflection of light of certain wavelengths (Nature 424, 852-855; 2003), creating bright colours that protect them from predators. Another example is a silver-coloured species of ant known as Cataglyphis bombycina, which is covered in dense arrays of triangular hairs that enhance solar reflection and thermal radiation, helping them survive in the harsh environment of the Sahara desert (Science $349,298-301 ; 2015)$. For my research, it was a particular species of beetle, Cyphochilus, that provided the necessary inspiration.

Cyphochilus is an Asian species of beetle that has bright white scales. This colouring is not the result of a white pigment, but is instead due to a disordered network of thin scales that can scatter incident light at all wavelengths (Science 315, 348; 2007). My supervisor, Andrea Fratalocchi, and I came up with the idea of reversing this effect and creating a novel black-body material. Such a material, which would be able to absorb light at all wavelengths and incident angles, could be created using a disordered distribution of nanostructures of a specific shape. The necessary nanostructures could, in particular, be formed by combining a nanorod and a nanosphere, with a sharp contact between them that enables broadband absorption. However, given their unusual geometry, synthesizing these structures proved to be a significant challenge.

Therefore, despite my enthusiasm for the fundamental idea, the project stalled because of the obstacles we faced in terms of nanostructure fabrication.

Jianfeng: I met Changxu at a social event organized by King Abdullah University of Science and Technology. As is typical at these colloquia, we began by talking about our own ongoing research projects and I heard about the problems Changxu was having in synthesizing particular nanostructures. I have a background in colloidal chemistry and knew that the basic

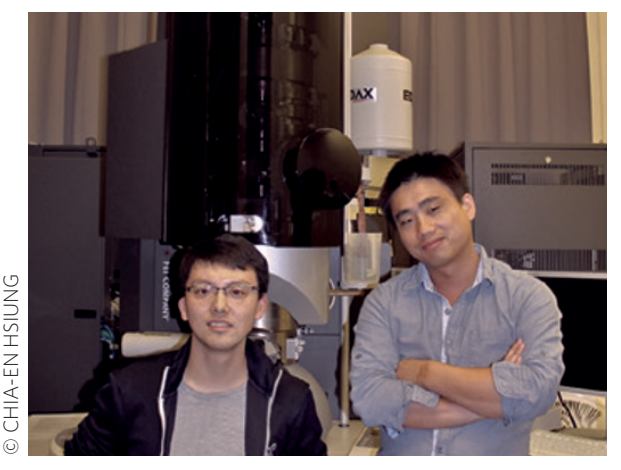

Changxu Liu (left) and Jianfeng Huang (right).

structures he needed - nanorods and nanospheres - could be synthesized using relatively straightforward chemical methods. I also suggested some ideas that could be potentially used to create the more intricate asymmetric dimer structures that were ultimately needed for the black-body system. After the conversation, we immediately decided to work together on the project.

The direct fabrication of asymmetric dimer structures is not easy. I discussed the issues with my advisor, Yu Han, and we decided that 'seeded growth', in which a single nanosphere is grown on a pre-synthesized nanorod, might be the solution. However, the seeded growth of monometallic dimers is not that simple either, because one of the major driving forces for dimerization is typically lattice mismatch. Therefore, when the growth material is identical to the seed material (as was required here), epitaxial growth on the entire surface of the seed is often favoured over the formation of a dimer. But with my expertise in colloidal nanocrystal design, combined with numerous attempts, I eventually discovered a thiol-based molecule that effectively switched the crystal growth modality from a continuous epitaxial shell to a discrete particle.

Once the nanostructures were successfully synthesized, we turned to their optical characterization. Though there was a short period of 'synchronization', it didn't take too long before we found an effective way to work together on this.

Changxu: After we had taken some optical measurements, I would analyse the data and discuss the results with Jianfeng to look to optimize the system. Through this process - the combined efforts of a physicist and chemist - we were able to develop the novel black-body system (the resulting paper is featured on page 60 of this issue) and create a new type of light source.

Jianfeng: Working in collaboration with Changxu, rather than on my own, definitely made it easier to perform a variety of tricky operations. I remember, for example, that characterizing the optical absorption of the samples required special care, but working together with Changxu on this made the operation very simple. Furthermore, distributing work, such as the data analysis, among us undoubtedly saved significant amounts of time and speeded up progress on the project.

Changxu: I really appreciate working in collaborations with chemists like Jianfeng, who can provide a totally different perspective on a problem. Here it allowed our designs to become a reality through specific knowledge on colloidal synthesis. For me personally, the experience was also valuable because it gave me the opportunity to work in a chemistry lab and learn more about a field outside my own, which has helped stimulate new ideas for future research.

CHANGXU LIU is a PhD student in the PRIMALIGHT group at King Abdullah University of Science and Technology, Thuwal 23955-6900, Saudi Arabia. JIANFENG HUANG recently completed his $\mathrm{PhD}$ at the Division of Physical Sciences and Engineering, King Abdullah University of Science and Technology, Thuwal 23955-6900, Saudi Arabia. e-mail: changxu.liu@kaust.edu.sa; jianfeng.huang@kaust.edu.sa

\section{Correction}

In the version of the In the Classroom article 'Graphic design for scientists' originally published (Nature Nanotech. 10, 1084; 2015), the name of the School in Karen Cheng's affiliation should have read 'School of Art, Art History and Design'. Corrected in the online versions after print 9 December 2015. 